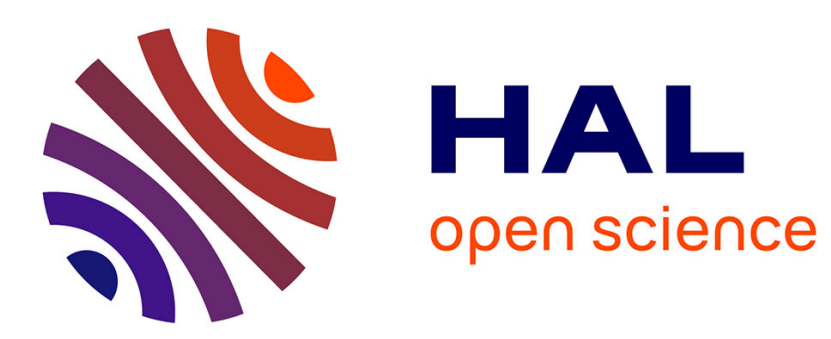

\title{
Martingalized Historical approach for Option Pricing
}

Christophe Chorro, Dominique Guegan, Florian Ielpo

\section{To cite this version:}

Christophe Chorro, Dominique Guegan, Florian Ielpo. Martingalized Historical approach for Option Pricing. Finance Research Letters, 2010, 7 (1), pp.24-28. 10.1016/j.frl.2009.11.002 halshs-00437927

\section{HAL Id: halshs-00437927 https://shs.hal.science/halshs-00437927}

Submitted on 2 Dec 2009

HAL is a multi-disciplinary open access archive for the deposit and dissemination of scientific research documents, whether they are published or not. The documents may come from teaching and research institutions in France or abroad, or from public or private research centers.
L'archive ouverte pluridisciplinaire HAL, est destinée au dépôt et à la diffusion de documents scientifiques de niveau recherche, publiés ou non, émanant des établissements d'enseignement et de recherche français ou étrangers, des laboratoires publics ou privés. 


\title{
Martingalized Historical approach for Option Pricing
}

\author{
Chorro C. ${ }^{*} \quad$ Guégan D. ${ }^{\dagger} \quad$ Ielpo F. ${ }^{\ddagger}$
}

November 3, 2009

${ }^{*}$ CES, MSE, 106 bd de l'hopital, 75013 Paris, France. Email: christophe.chorro@univ-paris1.fr

${ }^{\dagger}$ Corresponding author. CES, MSE, 106 bd de L'hopital, 75013 Paris, France. Email: dguegan@univ-paris1.fr. Tel: (33-1) 47-40-82-98. Fax: (33-1) 44-07-83-01.

${ }^{\ddagger}$ CES, MSE, 106 bd de l’hopital, 75013 Paris, France. Email: florianielpo@ensae.org 


\begin{abstract}
In a discrete time option pricing framework, we compare the empirical performance of two pricing methodologies, namely the affine stochastic discount factor (SDF) and the empirical martingale correction methodologies. Using a CAC 40 options dataset, the differences are found to be small: the higher order moment correction involved in the SDF approach may not be that essential to reduce option pricing errors. This paper puts into evidence the fact that an appropriate modelling under the historical measure associated with an adequate correction (that we call here a "martingale correction") permits to provide option prices which are close to market ones.
\end{abstract}

Keywords: Generalized Hyperbolic Distribution, Option pricing, Incomplete market, CAC40, Stochastic Discount Factor, Martingale Correction.

JEL classification: G12, G17, C5, C8. 


\section{Introduction}

Once the time series properties of financial asset's returns have been assessed, the biggest challenge to option pricing is to thoroughly select the right pricing measure. This article questions the importance of this step. We propose to answer this question by comparing two different modeling approaches: first, the affine Stochastic Discount Factor (SDF), as presented in Cochrane (2002) and then the Empirical Martingalization Correction (EMC) presented in Duan and Simonato (1998). The rationale behind this choice is the following: for the SDF framework, when moving from the historical distribution to the pricing one, each of the first four moments are changed. This is consistent with the existence of volatility trading and with the idea of skewness premia. In the second approach, the moment modification only involves the expectation, as in the Black and Scholes (1973)'s model. By comparing the results on a comparable ground with each methodology, we aim at answering the question of the real impact on option prices of the modifications of higher order moments when moving from the historical to pricing probability measure. This work is quite different in its philosophy of recent works focusing on a closed form SDF for pricing under the risk neutral measure such as Heston and Nandi (2000), Mercuri (2008) or Chorro et al. (2008a).

To achieve such a purpose, we use a time series model that properly fits the key known features of equity returns. The Nelson (1991)'s EGARCH model is used to handle the asymmetric reaction of the volatility to negative returns. To match the returns' leptokurticity and asymmetry, the conditional distribution of the innovations is assumed to be the Generalized Hyperbolic distribution. Using a CAC 40 dataset, we show that the option mispricing errors for both the SDF and EMC methodology are fairly closed: what seems to be the key to option pricing is more the precise modeling of the conditional distribution of the returns' innovations than the change in probability measure.

\section{The Modeling under $\mathbb{P}$}

Under the historical measure $\mathbb{P}$, the discrete time economy we consider is characterized by the time series dynamics of the stock price process $\left(S_{t}\right)_{t \in\{0,1, \ldots, T\}}$ given by

$$
Y_{t}=\log \left(\frac{S_{t}}{S_{t-1}}\right)=r+m_{t}+\sqrt{h_{t}} z_{t}, \quad S_{0}=s \in \mathbb{R}_{+},
$$

where $r$ is the risk free rate expressed on a daily basis and supposed to be constant and where $z_{t}$ is a sequence of independent and identically distributed centered random variables with variance 1 . 
In equation (1), we consider a general conditional time varying excess of return $m_{t}$ that depends on the constant risk premium $\lambda_{0}$. In practice, it will be fixed for the empirical study and we retain the following classical form:

$$
m_{t}=\lambda_{0} \sqrt{h_{t}}-\frac{1}{2} h_{t} .
$$

In order to capture asymmetry phenomena (such as leverage effects) observed in equity returns datasets, we restrict to the Nelson (1991)'s EGARCH(1,1) model:

$$
\log \left(h_{t}\right)=a_{0}+a_{1}\left(\left|z_{t-1}\right|+\gamma z_{t-1}\right)+b_{1} \log \left(h_{t-1}\right) .
$$

So as to deal with the leptokurticity and the asymmetry of the CAC 40 returns, the conditional distribution of the EGARCH model is chosen to be the Generalized Hyperbolic distribution. This distribution has been introduced by Barndorff-Nielsen (1977). It is driven by five parameters: $\lambda$ being a shape parameter, $\alpha$ controlling the kurtosis, $\beta$ controlling the skewness, $\delta$ and $\mu$ being respectively linked to the volatility and the expectation of the process.

For $(\lambda, \alpha, \beta, \delta, \mu) \in \mathbb{R}^{5}$ with $\delta>0$ and $\alpha>|\beta|>0$, the one dimensional $G H(\lambda, \alpha, \beta, \delta, \mu)$ distribution is defined by the following density function

$$
\begin{aligned}
& d_{G H}(x, \lambda, \alpha, \beta, \delta, \mu)=\frac{\left(\sqrt{\alpha^{2}-\beta^{2}} / \delta\right)^{\lambda}}{\sqrt{2 \pi} K_{\lambda}\left(\delta \sqrt{\alpha^{2}-\beta^{2}}\right)} \\
& \times e^{\beta(x-\mu)} \frac{K_{\lambda-1 / 2}\left(\alpha \sqrt{\delta^{2}+(x-\mu)^{2}}\right)}{\left(\sqrt{\delta^{2}+(x-\mu)^{2}} / \alpha\right)^{1 / 2-\lambda}},
\end{aligned}
$$

where $K_{\lambda}$ is the modified Bessel function of the third kind. For $\lambda \in \frac{1}{2} \mathbb{Z}$, the basic properties of the Bessel function allow to find simpler forms for the density. In particular, for $\lambda=1$, we get the Hyperbolic distribution whose $\log$-density is a hyperbola. For $\lambda=-\frac{1}{2}$, we obtain the Normal Inverse Gaussian distribution which is closed under convolution. This family has already been suggested as a model for financial price processes because its exponentially decreasing tails seem to fit some fundamental statistical behaviors of asset returns remarkably, as presented in Barndorff-Nielsen (1995) or Eberlein and Prause (2002). For instance, in Chorro et al. (2008b) with a similar dataset, the joint use of an EGARCH model with this GH distribution offers a very realistic assessment of the returns stochastic behavior. 


\section{Option pricing modeling}

Using the previous time series framework, we now discuss how to deal with the change in probability measure toward the risk neutral distribution. To serve our purpose, we consider two ways out of this problem. The first (the SDF approach) leads to a change in every moment of the distributions, whereas the second one (The EMC approach) states that the only difference between the risk neutral distribution and the historical one is the expectation.

\subsection{The Stochastic Discount Factor approach}

The approach that is briefly overviewed here received different presentations, the first one being reviewed in Cochrane (2002). The second one can be found in Gerber and Shiu (1994). The methodology unfolds as follows. The stochastic discount factor is the ratio of the risk neutral distribution to the historical one. We assume for the stochastic discount factor a particular parametric form: $\forall t \in\{0, \ldots, T-1\}$

$$
M_{t, t+1}=e^{\theta_{t+1} Y_{t+1}+\xi_{t+1}}
$$

where $Y_{t+1}=\log \left(\frac{S_{t+1}}{S_{t}}\right)$ and where $\theta_{t+1}$ and $\xi_{t+1}$ are $\mathcal{F}_{t}=\sigma\left(z_{u} ; 0 \leq u \leq t\right)$ measurable random variables. This hypothesis is implicit in many asset pricing models such as the Black and Scholes (1973)'s model.

We compute explicitly $\left(\theta_{t+1}, \xi_{t+1}\right)$. Considering the bond and the risky asset, the pricing relation

$$
P_{t}=E_{\mathbb{P}}\left[\Phi_{T} M_{t, T} \mid \mathcal{F}_{t}\right]
$$

with $\Phi_{T}$ the financial asset future random payoff, gives for $T=t+1$ the following restrictions for the stochastic discount factor

$$
\left\{\begin{array}{l}
E_{\mathbb{P}}\left[e^{r} M_{t, t+1} \mid \mathcal{F}_{t}\right]=1 \\
E_{\mathbb{P}}\left[e^{Y_{t+1}} M_{t, t+1} \mid \mathcal{F}_{t}\right]=1 .
\end{array}\right.
$$

Using the previous restrictions with Generalized Hyperbolic innovations, the distribution of $Y_{t}$ given $\mathcal{F}_{t-1}$ under $\mathbb{Q}$ is again a Generalized Hyperbolic one

$$
G H\left(\lambda, \frac{\alpha}{\sqrt{h_{t}}}, \frac{\beta}{\sqrt{h_{t}}}+\theta_{t}^{q}, \delta \sqrt{h_{t}}, M_{t}+\mu \sqrt{h_{t}}\right)
$$


with $M_{t}=r+m_{t}$ and where $\left(\theta_{t+1}^{q}, \xi_{t+1}^{q}\right)$ is the unique solution of (7). This essential property is proved in Chorro et al. (2008a) where the corresponding Monte Carlo pricing methodology is also explained.

\subsection{Empirical Martingale Correction approach}

This second approach to "risk neutralization" is inspired from the variance reduction tool presented in Duan and Simonato (1998). A similar idea used together with an optimization procedure is followed by Barone-Adesi et al. (2007) to overcome the classical option pricing framework. Here, we make no assumption on the shape of the pricing kernel and we compute prices for options with time to maturity $(T-t)$ by simulating sampled paths of the previous stochastic model under the historical distribution $\mathbb{P}$. To rule out arbitrage opportunities, we directly impose risk neutral constraints. The $i^{\text {th }}$ sampled historical final price for the underlying is denoted by $S_{T, i}=S_{t} \prod_{k=t+1}^{T} e^{Y_{k}}$ where $Y_{k}$ is given by (1) . The Empirical Martingale Correction works by replacing the previous sampled prices by:

$$
\tilde{S}_{T, i}=\frac{S_{T, i}}{1 / N \sum_{i=1}^{N} S_{T, i}} S_{t} e^{-r(T-t)}
$$

The sampled average of $\tilde{S}_{T, i}$ is exactly equal to $S_{t} e^{r(T-t)}$, that is the risk neutral conditional expectation. With this approach, we only shift the historical distribution in a way that prevents arbitrage opportunities by implicitly changing the drift of this distribution.

\section{Results}

The dataset that we use contains the following time series. We consider daily log returns of the French CAC 40 whose price at time $t$ is denoted $S_{t}$. The sample starts on January 2, 1988 and ends on October 26, 2007.

Concerning the estimation we are naturally led to a two steps methodology. We first estimate the EGARCH parameters driving the historical distribution with a Quasi Maximum Likelihood Estimation using a rolling window of 4'000 observations and ending at time $t$. At the second stage, since we exactly know the form of the density function of a GH distribution (7) we adopt a classical maximum likelihood approach to estimate the unknown remaining parameters $(\lambda, \alpha, \beta, \delta, \mu)$ using the residuals obtained at the previous stage. The key point in our approach is to maintain as much outliers in the dataset as possible: these extremal events are essential to fit the GH parameters and to control the tail behaviors. 
The average values obtained for each parameters driving the EGARCH model are presented in table 1 and those driving the GH distribution are presented in 2 . Then, we price options using either the SDF or the EMC methodologies.

We get the approximated option price $\tilde{C}($.$) as the sample average of the simulated final prices:$

$$
\tilde{C}(t, T, K)=\frac{1}{N} \sum_{i=1}^{N}\left(S_{T, i}-K\right)_{+} e^{-r(T-t)} .
$$

In practice, the number of sampled paths $N$ is equal to 10000 .

Finally, to compare the two option pricing methods, we use the average absolute relative pricing errors criterion for the working days $t$ between January 2, $2006\left(\tau_{1}\right)$ and October 26, $2007\left(\tau_{2}\right)$. Let $\tilde{C}\left(t, T_{j}, K_{i}\right)$ be the estimated call option price with a time to maturity equal to $T_{j}-t$ and a strike price worth $K_{i}$ and $C\left(t, T_{j}, K_{i}\right)$ be the corresponding quoted market option price. Then the criterion is

$$
A A R P E=\sum_{t=\tau_{1}}^{\tau_{2}} \sum_{j=1}^{J_{t}} \sum_{i=1}^{G_{t, j}}\left|\frac{\tilde{C}\left(t, T_{j}, K_{i}\right)-C\left(t, T_{j}, K_{i}\right)}{C\left(t, T_{j}, K_{i}\right)}\right|,
$$

where $J_{t}$ is the number of call option maturities $T_{j}$ available at time $t$ and $G_{t, j}$ the number of strikes $K_{i}$ available at time $t$ for this particular maturity $T_{j}$.

This criterion is robust to the well known fact that option pricing errors are proportional to the moneyness: out of the money call option prices are very low, and so are the usual errors found. The converse is true for deep in the money option prices. Table 3 presents the value obtained for this criterion with the two different approaches to produce this change in distribution.

First, the scale of errors obtained are very closed to the one obtained in Barone-Adesi et al. (2007), whereas they directly optimize the GARCH parameters to match the option prices. Second, for time to maturities beyond 1 year, the pricing kernel based option prices are clearly dominated by the simpler method. Third, for time to maturities between .5 and 1 year, the results get trickier to analyze: depending on the moneyness, one method may dominate the other. Globally, the errors obtained for each approach are very close. The main conclusion of these estimation results can be summarized as follows: when taking into account the returns' time series properties with much care, the change in distribution from the historical to the risk neutral probabilities is not that essential anymore. The simple martingalization produced by the EMC approach is enough to ensure small pricing errors. 


\section{Conclusion}

We compare two different approaches to price options in a discrete time setting. The first one lies on classical theoretical results derived from the Black and Scholes (1973)'s hedging methodology to change from the historical to the risk neutral distribution. The second one is only based on no arbitrage requirements avoiding any technical arguments. By doing this, we end up with the intuition that a simple change in the drift clearly challenges a more sophisticated methodology that is widely used in the financial economics literature. The use of a time series model with innovations that encompasses most of the known features of financial assets' returns seems to be more essential to option pricing.

A forthcoming paper is ongoing to deeply explore this feature with other datasets and also to compare the (EMC) to the pricing methodology proposed by Elliott and Madan (1998).

\section{References}

Barndorff-Nielsen, O. (1977). Exponentially Decreasing Distributions for the Logarithm of Particle Size. Proc. Roy. Soc. London A, (353):401-419.

Barndorff-Nielsen, O. (1995). Normal Inverse Gaussian Processes and the Modeling of Stock Returns. Research Report 300, Department Theoretical Statistics, Aarhus University.

Barone-Adesi, G., Engle, R. F., and Mancini, L. A. (2007). GARCH Option Pricing Model in Incomplete Markets. to appear in the Review of Financial Studies.

Black, F. and Scholes, M. (1973). The Pricing of Option and Corporate Liabilities. Journal of Political Economy, (81):637-654.

Chorro, C., Guégan, D., and Ielpo, F. (2008a). Option Pricing Under GARCH Models with Generalized Hyperbolic Innovations (I): Theoretical Results. Centre d'Economie de la Sorbonne Working Paper.

Chorro, C., Guégan, D., and Ielpo, F. (2008b). Option Pricing Under GARCH Models with Generalized Hyperbolic Innovations (II): Empirical Results. Centre d'Economie de la Sorbonne Working Paper.

Cochrane, J. (2002). Asset Pricing. Princeton University Press.

Duan, J. and Simonato, J. (1998). Empirical Martingale Simulation for Asset Prices. Management Science, (44):1218-1233. 
Eberlein, E. and Prause, K. (2002). The Generalized Hyperbolic Model: Financial Derivatives and Risk Measures. In: H. Geman, D. Madan, S. Pliska, T. Vorst (Eds.), Mathematical Finance-Bachelier Congress 2000, Springer Verlag, pages 245-267.

Elliott, R. and Madan, D. (1998). A Discrete Time Equivalent Martingale Measure. Mathematical Finance, 2(8):127-152.

Gerber, H. and Shiu, S. (1994). Option Pricing by Esscher Transforms. Transaction of Society of Actuaries, a(46):99-191.

Heston, S. and Nandi, S. (2000). A Closed-Form GARCH Option Valuation. The Review of Financial Studies, (13):585-625.

Mercuri, L. (2008). Option pricing in a garch model with tempered stable innovations. Finance Research Letters, 5(3):172-182.

Nelson, D. (1991). Conditional Heteroskedasticity in Asset Returns. Econometrica, (59):347-370. 


\begin{tabular}{cccccc}
\hline \hline & $a_{0}$ & $b_{1}$ & $a_{1}$ & $\gamma$ & $\lambda_{0}$ \\
\hline \hline Average & -5.51 & 0.47 & 0.01 & 0.91 & 0.08 \\
Std. Dev. & 0.33 & 0.04 & 0.02 & 0.02 & 0.03 \\
\hline \hline
\end{tabular}

Table 1: Descriptive statistics on the average estimated parameters for the EGARCH model.

\begin{tabular}{cccccc}
\hline \hline & $\alpha$ & $\beta$ & $\delta$ & $\mu$ & $\lambda$ \\
\hline \hline Average & 308.42 & -2.77 & 335.64 & 3.04 & -78.46 \\
Std. Dev. & 139.90 & 5.08 & 152.02 & 5.51 & 76.68 \\
\hline \hline
\end{tabular}

Table 2: Descriptive statistics on the average estimated parameters for the GH distribution.

\begin{tabular}{|c|c|c|c|c|c|c|}
\hline & \multicolumn{6}{|c|}{$\mathbf{. 5}<$ Maturity $<\mathbf{1}$} \\
\hline & & $\overline{[.8-.9]}$ & {$[.9-1]$} & [1-1.1] & $\overline{[1.1-1.2]}$ & $>1.2$ \\
\hline SDF correction & Nelson GH & 0.072508156 & 0.034220274 & 0.08973236 & 0.211844412 & 0.473990647 \\
\hline Mart. Correction & Nelson GH & 0.070375688 & 0.032051441 & 0.112397312 & 0.225184538 & 0.40081137 \\
\hline & \multicolumn{6}{|c|}{ Maturity $>1$} \\
\hline & & [.8-.9] & {$[.9-1]$} & [1-1.1] & [1.1-1.2] & $>1.2$ \\
\hline SDF correction & Nelson GH & 0.120757162 & 0.088165393 & 0.069513071 & 0.118837264 & 0.261635523 \\
\hline Mart. correction & Nelson GH & 0.119581221 & 0.087505694 & 0.067820071 & 0.119243876 & 0.266680359 \\
\hline
\end{tabular}

Table 3: Absolute average mispricing errors for the CAC 40 french index disaggregated by maturities and moneyness, using the stochastic discount factor restrictions (SDF correction) or the empirical martingale correction (Mart correction). 